\title{
Chain Inflation Reconsidered
}

\author{
James M. Cline ${ }^{*}$ Guy D. Moore † Yi Wang ${ }^{\ddagger}$ \\ Physics Department, McGill University, Montreal, H3A2T8, Canada
}

\begin{abstract}
We investigate density perturbations in the chain inflation scenario, where the inflaton undergoes successive tunneling transitions along one field direction. First we show that when the bubble walls associated with such a phase transition meet, they induce the next phase transition and continue to propagate as phase interfaces. Then we present an analytical calculation of the density fluctuations and an estimate of non-Gaussianities such as $f_{N L}$, which we find to be small (of order 1). To get the right amplitude for the power spectrum, there have to be $10^{8}$ phase transitions per Hubble time, a significant model building challenge. We find that working models of chain inflation must be rather strongly coupled, and thus have a very limited range of validity as effective field theories. We discuss generalizations to the multiple field case, the curvaton scenario, and noncanonical kinetic terms.
\end{abstract}

\section{Introduction}

In a scenario dubbed "chain inflation" [1, 2] it was suggested that the dynamics of inflation may be dominated by a series (chain) of phase transitions, each induced by the quantum nucleation of bubbles (tunneling events). Provided that each phase transition occurs much less than one Hubble time after the previous one, this scenario is free from the problem of "old inflation" [3] that the space still in the previous phase continues to inflate and prevents the completion of a phase transition. The need for several phase transitions per Hubble time, and the requirement that inflation persist for at least $\sim 60$ Hubble times to solve the flatness and horizon problems, means that the scenario requires a potential with a large number of minima, tunneled through in sequence. This is a challenge for model building but not an insurmountable one.

The density perturbations arising from chain inflation have been investigated in 4,5 , 6]. A simplified model of chain inflation was proposed in [7]. However, in these works either the density fluctuation has to be determined numerically, or features of standard

\footnotetext{
*jcline@hep.physics.mcgill.ca

†guymoore@hep.physics.mcgill.ca

‡wangyi@hep.physics.mcgill.ca
} 
slow roll inflation (with vacuum fluctuations instead of fluctuations from tunneling) are assumed in the calculation. Thus an analytical calculation for the perturbation theory is still absent for chain inflation.

In this paper we demonstrate three properties of chain inflation which seem not to have been addressed, and which are each troubling from the point of view of building realistic models. First, when the chain of tunneling events takes place in a single field direction, the collision of two bubble walls will trigger a nucleation of the next phase. Second, the rate at which the field, responsible for the chain of phase transitions, moves along its potential is controlled by the rate of bubble nucleations. But since bubble nucleations are stochastic in nature, the fluctuations in the field value, which become curvature perturbations, are controlled by the Poisson statistics of nucleation events. To meet current observational limits on curvature perturbations requires of order $10^{8}$ phase transitions per Hubble time during the observable part of inflation, requiring a potential with of order $10^{10}$ or more local minima to tunnel through successively. The third problem is that it is challenging to provide a potential where the nucleation rate between minima is large enough to support chain inflation, in a field theory that remains valid to scales that are much higher than the mass of the field.

Our paper is organized as follows: in Section 2, we set up our model and discuss the dynamics of bubble collisions which are essential to understanding the overall evolution of the inflaton. In section 3 we compute the time dependence of the mean field which constitutes the homogeneous and isotropic background. In Section 4, we consider the density perturbations and we calculate the power spectrum analytically, showing that it takes a simple form. Section 5 investigates the simplest explicit realization of a chain inflation model and identifies a narrow region of parameter space in which it satisfies observational and internal consistency requirements. In Section 6, we consider threeand higher-point correlations. We consider generalizations in Section 7 and conclude in Section 8 ,

\section{Setup and bubble collision dynamics}

We consider a simple model of chain inflation, with a single scalar field whose potential is a combination of an oscillation and a slope. Specifically, we consider

$$
V(\varphi)=V_{0}(\varphi)+V_{1} \sin (\omega \varphi)
$$

where $V_{0}$ is a slowly varying potential and $V_{1}, \omega$ are constants. A potential of this kind is illustrated in figure 1. In practice the pertinent features of the potential are that each minimum is lower than the last, and that the nucleation rate between each pair of minima is approximately the same.

We will also assume that the mean spacetime separation between nucleation events is large compared to the size of a bubble at nucleation. Otherwise, a description in terms of bubble nucleations does not make sense. Since nucleation events must in turn be common on the Hubble scale, this ensures that the instanton method [8, 9] applies. We 


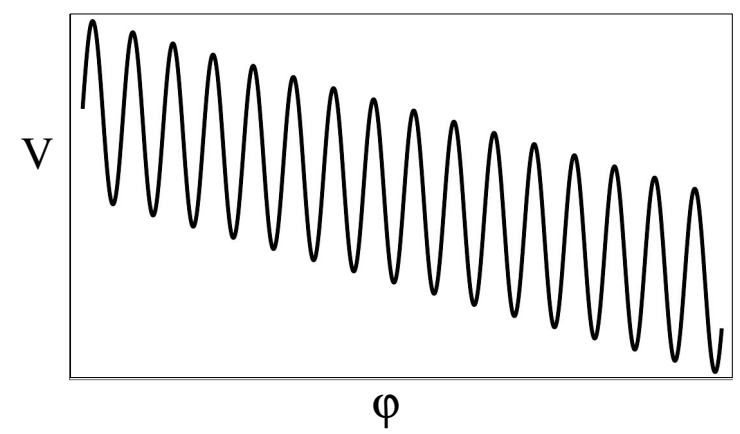

Figure 1: A sample potential for single-field chain inflation, as in equation (1).

will also assume that most of the energy from the potential drop between minima goes into accelerating the bubble wall.

Unlike the case in [8, 9], there is a chain of metastable vacua. As we now argue, in this case collisions of bubble walls trigger the subsequent phase nucleation. (This phenomenon has been previously discussed in ref. [10].) Specifically, numbering the potential minima from high to low as $\ldots, 1,2,3, \ldots$, when an interface between minima 1 and 2 meets another interface between 1 and 2, they pass through each other and become interfaces between minima 2 and 3 . To see why, note first that the walls become very relativistic and Lorentz contract to much narrower than the "natural" width set by the mass (second derivative of the potential). The process of walls crossing therefore occurs on length and time scales shorter than the natural scale in the potential. Therefore it is controlled by the kinetic term. Scalar field dynamics governed by only the kinetic term is free field theory, which is solved by the linear wave equation. According to the linear wave equation, when two interfaces meet, in which the field changes from value $\varphi_{0}$ to value $\varphi_{0}+2 \pi / \omega$, they pass through each other, turning into interfaces where $\varphi$ changes from $\varphi_{0}+2 \pi / \omega$ to $\varphi_{0}+4 \pi / \omega$. This is precisely the same behavior as colliding kinks in the sine-Gordon model.

These remarks apply both in our case, and to the case of a potential with only two minima. When there are only two minima, the region between the bubble walls is displaced from the potential minimum and the field there oscillates, with a time scale set by the parameters of the potential. These oscillations exhaust the energy of the bubble walls, which slow and stop, completing the transition. But in our case, the new value of the field after the walls have crossed is again a minimum of the potential, and the field stays there. After passing each other, the walls are again interfaces connecting a higher to a lower minimum, and they continue to accelerate. We have verified these statements by simulating wave interactions in $1+1$ dimensional classical field theory using the potential from Eq. (1). Some snapshots of the field configuration as two walls meet are provided in Figure 2 as an illustration.

Since the bubble walls do not stop or dissipate their energy when they collide, no thermal radiation is created by bubble collisions. Thus we do not need to worry about the perturbations and back-reaction from the radiation components created by the wall 


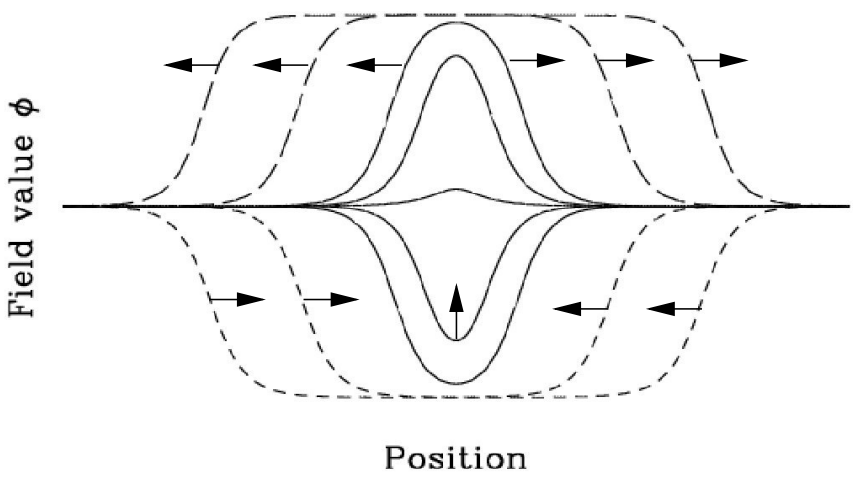

Figure 2: Collision of bubble walls. Short-dashed lines show $\varphi$ at two moments before the walls meet; solid lines show $\varphi$ at a series of times as the walls meet; long dashes show two times after the walls have crossed. When the walls cross, they switch from connecting minima 1 and 2, to connecting minima 2 and 3.

collisions. Since energy in bubble walls redshifts like radiation 11 they also do not come to dominate the energy density of the system.

However, there is another complication of nucleation in a potential with multiple minima such as that given in Eq. (1). When the "slope" is relatively large compared to the "oscillation," quantified by the parameter $B \equiv 2 \pi V_{0}^{\prime} / V_{1} \omega$, not all of the potential energy liberated by a bubble wall goes into accelerating the wall. Some goes into creating ripples in the $\varphi$ field, as illustrated in Figure 3, which shows moving bubble wall profiles for $B=3,4,5,6$. Solving the field equations for a single accelerating bubble wall using the potential of Eq. (1), we find that for $B>6.02$ these ripples grow large enough that they overshoot the next barrier and induce the next phase transition. This sets off a cascade in which $\varphi$ "runs away" down the potential. So $B<6.02$ is required to ensure that bubble nucleations only induce a single phase transition and do not set off a catastrophic run down the potential. This puts a lower limit on the potential barrier relative to the potential drop between minima. (Note that at $B=2 \pi$ the minima disappear; so the limiting value of $B$ is quite close to where the potential becomes monotonic.)

This requirement on the potential has consequences for the bubble nucleation action. Applying the procedure of Coleman et al. [8, 9] to find the nucleation action $S$ for the potential in Eq. (1) (without making any thin-wall approximation), we find the result depends only on $B$ and $V_{1} \omega^{4}: S=\left(V_{1} \omega^{4}\right)^{-1} F(B)$, where the function $F$ is fit by $F=(2 \pi)^{4}\left(348.6 / B^{3}-0.738 / B^{2}-8.44 / B\right)$ on the interval $1 \lesssim B \lesssim 6$. In particular, at the critical value $B=6.02$, we find that $F=333.5$. (For smaller values of $B, F$ is even larger, so this represents the best case for getting a large tunneling rate.) The nucleation

\footnotetext{
${ }^{1}$ this can be most easily established by writing the stress-energy tensor for a bubble wall with tension $\epsilon$ at rest, $T_{\mu \nu}=\operatorname{diag}[\epsilon, 0,-\epsilon,-\epsilon]$ and then boosting by a large factor for a bubble moving near the speed of light. The diagonal part after boosting is $T_{\mu \nu}=\epsilon \gamma^{2} \operatorname{diag}\left[1, v^{2},-\gamma^{-2},-\gamma^{-2}\right]$ from which it follows that the pressure is $p=\frac{1}{3}\left(\gamma^{2} v^{2}-2\right) \epsilon \cong \gamma^{2} \epsilon / 3=\rho / 3$.
} 


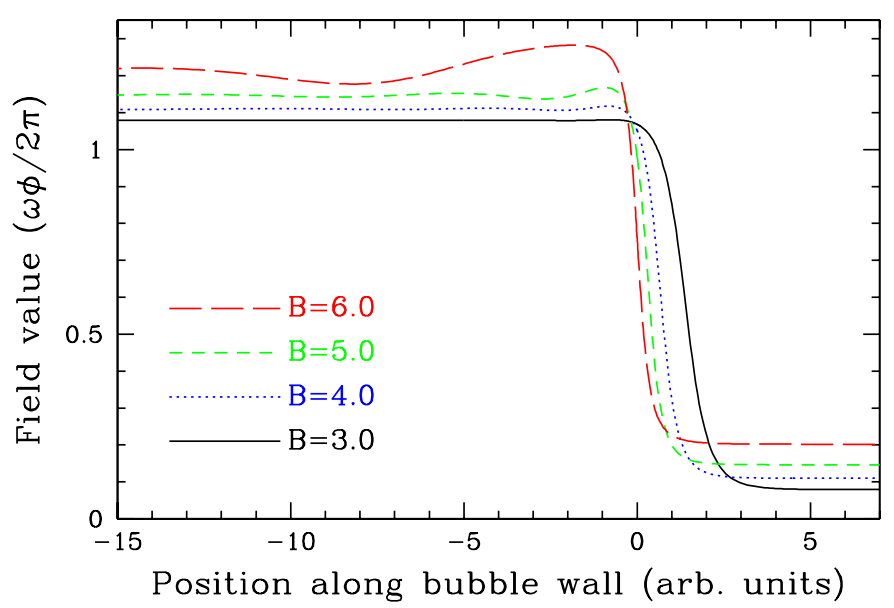

Figure 3: profile of a bubble wall, moving towards the right, for several values of $B$ the ratio of "slope" to "oscillation" heights in the potential. The larger the slope, the larger the "ripples" which appear behind the wall. For a critical value of $B$ just larger than the largest shown, the ripples nucleate the next transition.

action can only be made small by choosing a large value for $V_{1} \omega^{4}$. But this quantity is also the maximal value of the fourth derivative of the potential. Provided that the potential can be regarded as the effective potential of a canonically normalized scalar field in a four-dimensional quantum field theory, the potential is only stable against large loop corrections if $V^{\prime \prime \prime \prime} \sim V_{1} \omega^{4}$ is small enough ${ }^{2}$ say, $V^{\prime \prime \prime \prime}<2 \pi$. Imposing this condition, we find $S \geq 53$. This leads to a bubble nucleation rate $\Gamma \sim \exp (-S)$ which may be too small to allow the phase transitions to complete in a Hubble time. We will see that this requirement makes it difficult, though not impossible, to find consistent models of chain inflation.

\section{Mean evolution of the field}

We now proceed to study how the mean field $\langle\varphi\rangle$ evolves in chain inflation, which is quite different from the slow-roll dynamics of conventional inflation models. We work in comoving coordinate $x$ and conformal time $\tau$. In these coordinates, light cones are straight lines with slope $45^{\circ}$. The tunneling rate can be written as a function of time as

$$
\Gamma_{c}=\frac{\Gamma}{a^{4}}=\frac{\Gamma}{H^{4} \tau^{4}},
$$

where $\Gamma_{c}$ is the tunneling rate in comoving coordinates and conformal time, and $\Gamma$ is the tunneling rate in physical coordinates. We assume $\Gamma$ is a constant, or varying slowly

\footnotetext{
${ }^{2}$ If we consider a potential with $V^{\prime \prime \prime \prime} \gg 2 \pi$, defined at some physical scale $\mu_{1}$, then the potential as measured at a more infrared scale $\mu_{2}$ will have a smaller value $V^{\prime \prime \prime \prime} \lesssim 2 \pi / \ln \left(\mu_{1} / \mu_{2}\right)$. The condition $V^{\prime \prime \prime \prime} \lesssim 2 \pi$ is therefore unavoidable if we demand that the theory exist at an energy scale a few times higher than the mass scale of the potential $\sim V_{1} \omega^{2}$.
} 
along the chain of meta-stable vacua ${ }^{3}$

To model the phase transition dynamics, a random variable $X(x, \tau)$ is placed on every spacetime point, denoting the probability density for a spontaneous (in other words, not from bubble wall collision) tunneling event to take place. As we discussed, the collision of bubble walls does not stop the propagation of a bubble wall; so any spontaneous nucleation event along the past light-cone of some spacetime point $(x, \tau)$ will cause a bubble wall to induce a phase transition at $(x, \tau)$, and any spontaneous nucleation event inside the past light cone of $(x, \tau)$ has caused such a transition along the past world-line of an observer at $(x, \tau)$. Therefore the scalar field value at a point $(x, \tau)$ is determined by a sum over all spontaneous nucleation events in the past light cone. We find

$$
\varphi(x, \tau)=\Delta \varphi \int^{\tau} d \tau^{\prime} \int d^{3} x^{\prime} \Theta\left[\left(\tau-\tau^{\prime}\right)^{2}-\left(x-x^{\prime}\right)^{2}\right] X\left(x^{\prime}, \tau^{\prime}\right),
$$

where $\Delta \varphi \equiv 2 \pi / \omega$ is the distance between neighboring minima in field space, and the integral with the Heaviside $\Theta$ function is over the interior of the past light cone of $(x, \tau)$. The random variables $\{X(x, \tau)\}$ obey the Poisson distribution

$$
X \sim \operatorname{Pois}\left(\Gamma_{c} \mathrm{~d}^{3} x \mathrm{~d} \tau\right)=\text { Pois }\left(\frac{\Gamma \mathrm{d}^{3} x \mathrm{~d} \tau}{H^{4} \tau^{4}}\right) .
$$

Note that the sum of Poisson random variables is also a Poisson random variable, with

$$
X \sim \operatorname{Pois}\left(\mu_{X}\right), \quad Y \sim \operatorname{Pois}\left(\mu_{Y}\right) \quad \Rightarrow \quad X+Y \sim \operatorname{Pois}\left(\mu_{X}+\mu_{Y}\right) .
$$

Thus the summation over the past light cone (analog of $\mu_{X}+\mu_{Y}$ in equation (5p) takes the form

$$
\int_{\tau_{i}}^{\tau} \mathrm{d} \eta \frac{4}{3} \pi(\tau-\eta)^{3} \frac{\Gamma}{H^{4} \eta^{4}}=\frac{\varphi_{i}}{\Delta \varphi}+\frac{4 \pi \Gamma}{3 H^{4}} \log \left(\frac{\tau_{i}}{\tau}\right)=\frac{\varphi_{i}}{\Delta \varphi}+\frac{4 \pi \Gamma}{3 H^{3}}\left(t-t_{i}\right),
$$

where the constant $\varphi_{i}$ is the field value at an initial time $\tau_{i}$, and $t$ denotes proper times of a comoving observer such that $d t=a d \tau$. Here we have used the approximation $\left|\tau_{i}\right| \gg|\tau|$. Because of the exponential expansion of space, this approximation should hold for any reasonably early $\tau_{i}$. Equation $(6)$ means that $\varphi(x, \tau)$ obeys the distribution

$$
\varphi(x, \tau) \sim \Delta \varphi \times \text { Pois }\left(\frac{\varphi_{i}}{\Delta \varphi}+\frac{4 \pi \Gamma}{3 H^{3}}\left(t-t_{i}\right)\right) .
$$

From properties of the Poisson distribution $(\langle X\rangle=\mu$ for $X \sim \operatorname{Pois}(\mu))$, the expectation value of $\varphi(x, \tau)$ is

$$
\langle\varphi(x, \tau)\rangle=\varphi_{i}+\frac{4 \pi \Gamma \Delta \varphi}{3 H^{3}}\left(t-t_{i}\right)
$$

\footnotetext{
${ }^{3}$ If $\Gamma$ varies significantly in one Hubble time, the perturbation spectrum will have a large tilt, in contradiction to observations.
} 


\section{The power spectrum}

In this section, we first calculate the position space two point function and then Fourier transform to get the power spectrum. Before doing so, it is helpful to first have an intuitive understanding of the fluctuations. As illustrated in figure 4 , bubble walls move nearly at the speed of light, and the collision of bubble walls does not affect their dynamics. When there is a fluctuation at an initial slice (denoted by $a$ and $b$ ), it propagates at the speed of light to $a^{\prime}$ and $b^{\prime}$ at a later slice. Since fluctuations cannot propagate very far in the comoving diagram, they are conserved on super-Hubble scales.

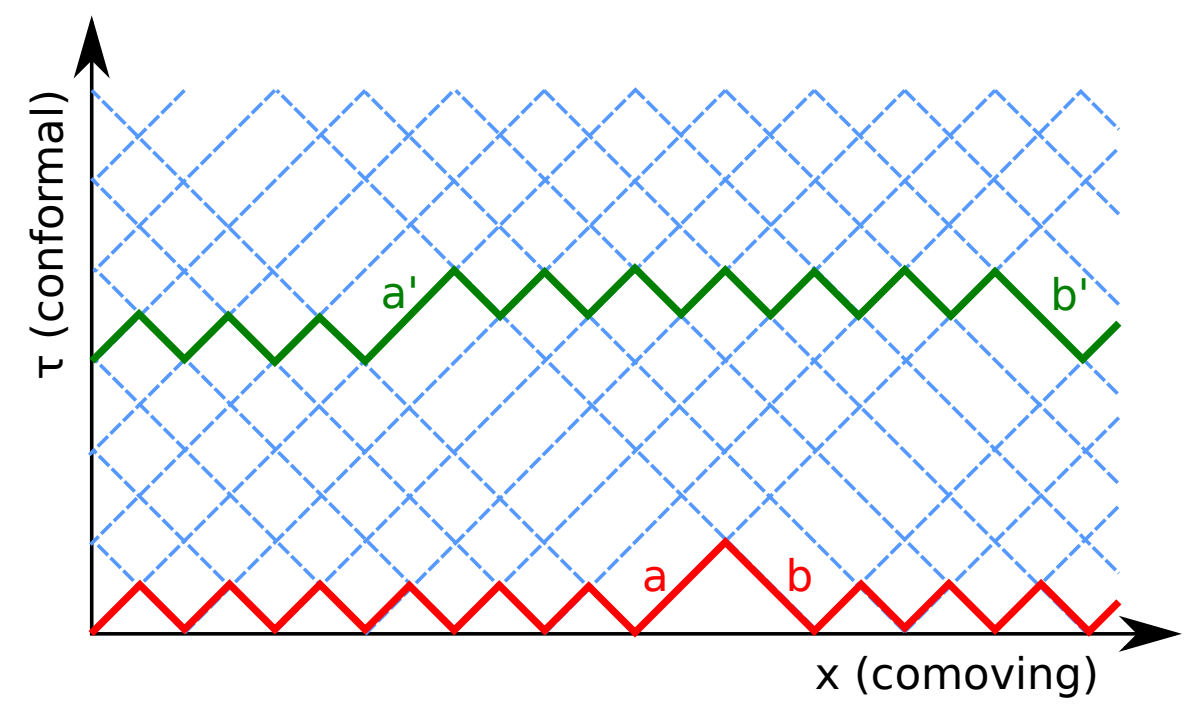

Figure 4: The propagation of fluctuations for single field chain inflation. The lower heavy (red) line denotes an initial slice. The fluctuation on this initial slice is propagated to later slices, for example the one denoted by upper heavy (green) line, and becomes conserved on super-Hubble scales.

To calculate the position-space two-point correlation $\langle\delta \varphi(x, \tau) \delta \varphi(x+r, \tau)\rangle$, we note that $\varphi(x, \tau)$ and $\varphi(x+r, \tau)$ can be expressed in terms of equation (3). In the overlapping region between the past light cone of $(\mathbf{x}, \tau)$ and $(\mathbf{x}+\mathbf{r}, \tau)$, the random variables in the summation for $\varphi(x, \tau)$ and $\varphi(x+r, \tau)$ are identical. On the other hand, outside the overlapping region, the random variables in the summation for $\varphi(x, \tau)$ and $\varphi(x+r, \tau)$ are independent. The latter results in zero expectation value for correlations, and the former leads to the connected part of the two-point function

$$
\langle\delta \varphi(x, \tau) \delta \varphi(x+r, \tau)\rangle_{c}=\Delta \varphi^{2} \int_{-\infty}^{\tau} \mathrm{d} \eta V_{2}(\eta) \frac{\Gamma}{H^{4} \eta^{4}},
$$

where

$$
V_{2}(\eta) \equiv \begin{cases}\frac{4}{3} \pi(\tau-\eta-r / 2)^{2}(\tau-\eta+r / 4) & \text { when } r<2(\tau-\eta) \\ 0 & \text { when } r \geq 2(\tau-\eta)\end{cases}
$$


is the intersection volume of the two past light cones at time $\eta 4^{4}$ Here we have put the start of chain inflation at $\tau_{i}=-\infty$. In other words we assume chain inflation begins several e-folds before the largest currently observable scales exit the horizon. Otherwise there can be modifications to the lowest observable multipoles, which depend on the details of what happens before chain inflation, an issue we do not want to address here.

The integration in equation (9) has an IR divergence, proportional to $\log \tau$. The IR divergence is a characteristic of random walk behavior in position space, which is also present in standard slow roll inflation. We do not need to worry about this divergence because it is absent after the Fourier transformation. The Fourier space two-point correlation function is given by

$$
\left\langle\delta \varphi_{\mathbf{k}_{1}}(\tau) \delta \varphi_{\mathbf{k}_{1}}(\tau)\right\rangle=(2 \pi)^{3} \delta^{3}\left(\mathbf{k}_{1}+\mathbf{k}_{2}\right) \int_{0}^{\infty} \mathrm{d} r \frac{4 \pi r \sin (k r)}{k}\langle\delta \varphi(0, \tau) \delta \varphi(r, \tau)\rangle .
$$

Inserting equation (9) into (11), interchanging the order of integration, and then taking the limit $k \equiv k_{1}=k_{2} \ll-1 / \tau$ to consider the super-Hubble modes, we obtain

$$
\left\langle\delta \varphi_{\mathbf{k}_{1}}(\tau) \delta \varphi_{\mathbf{k}_{1}}(\tau)\right\rangle=(2 \pi)^{3} \delta^{3}\left(\mathbf{k}_{1}+\mathbf{k}_{2}\right) \frac{8 \pi^{3} \Gamma \Delta \varphi^{2}}{3 H^{4} k^{3}} .
$$

Using the relation

$$
\zeta=-\frac{H}{\dot{\varphi}} \delta \varphi
$$

where $\zeta$ is the curvature perturbation in the uniform energy density slice, and the time derivative of equation (8), we have

$$
\left\langle\zeta_{\mathbf{k}_{1}} \zeta_{\mathbf{k}_{2}}\right\rangle=(2 \pi)^{3} \delta^{3}\left(\mathbf{k}_{1}+\mathbf{k}_{2}\right) \frac{3 \pi H^{4}}{2 \Gamma k^{3}} .
$$

Comparing to the relation

$$
\left\langle\zeta_{\mathbf{k}_{1}} \zeta_{\mathbf{k}_{2}}\right\rangle=(2 \pi)^{3} \delta^{3}\left(\mathbf{k}_{1}+\mathbf{k}_{2}\right) \frac{2 \pi^{2}}{k^{3}} P_{\zeta}
$$

gives the normalization of the power spectrum,

$$
P_{\zeta}=\frac{3 H^{4}}{4 \pi \Gamma} .
$$

Inserting the observed value $P_{\zeta}=2.42 \times 10^{-9}$, we find one of our main results, that $\Gamma / H^{4} \simeq 10^{8}$. The number of tunnelings per unit Hubble time and volume has to be surprisingly large.

Our result disagrees with previous computations of the normalization of the power spectrum [4, 5], whose validity was assumed by subsequent papers such as ref. [11. Our different finding is in part due to the fact that previous references did not consider

\footnotetext{
${ }^{4}$ We used the properties $\langle X\rangle=\mu,\left\langle X^{2}\right\rangle=\mu^{2}+\mu$ of the distribution Pois $(\mu)$ to derive this result.
} 
bubble nucleations triggered by bubble wall collisions. Our methodologies have further differences; ref. [4] calculated the spectrum numerically and obtained $P_{\zeta} \sim\left(H^{4} / \Gamma\right)^{5 / 3}$ in contrast to our result $P_{\zeta} \sim\left(H^{4} / \Gamma\right)$. Ref. [5] obtained the conventional result $P_{\zeta} \sim$ $H^{2} /\left(M_{p}^{2} \epsilon\right)$ where $\epsilon=-\dot{H} / H^{2}$ is the Hubble slow roll parameter. This arises from assuming vacuum initial conditions to solve the equation of motion for the perturbations, which is not valid for chain inflation.

The spectral index is

$$
n_{s}-1=\frac{\mathrm{d} \log P_{\zeta}}{\mathrm{d} \log k}=4 \frac{\dot{H}}{H^{2}}-\frac{\dot{\Gamma}}{H \Gamma}
$$

When $\dot{\Gamma}$ is negligible, the spectrum is necessarily red since $\dot{H}<0$. However, $\dot{\Gamma} / H \Gamma$ depends on how $V_{0}, V_{1}$ vary with $\varphi$, with indeterminate sign and magnitude. Therefore chain inflation does not make a definite prediction for the spectral tilt; it can generate a large tilt of either sign. In particular, since we saw that $\Gamma$ is extremely exponentially sensitive to $B$ the ratio of slope to oscillation height in the potential, the slightest evolution of $B$ along the potential will significantly change $\Gamma$. It appears to require a carefully tuned potential to ensure that the spectral tilt is not large.

\section{Explicit realizations}

In this section we investigate the extent to which one can find simple working models of chain inflation, taking into account all the phenomenological constraints and requirements of consistency. We take the simplest possible potential of the form (1), with

$$
V(\varphi)=V_{0}-A \varphi+V_{1} \sin (\omega \varphi)
$$

where $V_{0}$ is constant and $A>0$. This form is assumed to hold during inflation; for $\varphi$ exceeding some maximum value $\varphi_{\text {end }}, V_{0}$ must become field-dependent and reach a minimum in order for inflation to end. Motivated by the discussion in section 2, we will take the optimal value for $B$,

$$
B \equiv \frac{2 \pi A}{V_{1} \omega}=6.02
$$

so as to alleviate as much as possible the tension between having a large enough tunneling rate and a small enough value of the effective self-coupling of the field,

$$
\lambda=\max V^{\prime \prime \prime \prime}=V_{1} \omega^{4}
$$

We further assume that $V_{0} \gg V_{1},|A \varphi|$ so that the Hubble parameter is approximately constant during inflation, $H \cong \sqrt{V_{0} /\left(3 M_{p}^{2}\right)}$.

To estimate the tunneling rate, we use 12

$$
\Gamma \sim S^{2} M^{4} e^{-S}
$$


where $S$ is the action of the bounce solution, and $M$ is the mass scale from the fluctuation determinant, which we estimate using the curvature of the potential, $M^{2} \sim V_{1} \omega^{2}$. The bounce action was already computed in section 2, $S=333.5 / \lambda$. Using (21) and $H \cong$ $\sqrt{V_{0} /\left(3 M_{p}^{2}\right)}$ we can express the requirement $\Gamma / H^{4}=10^{8}$, coming from the normalization of the power spectrum, in terms of the model parameters:

$$
9 M_{p}^{4} V_{0}^{-2} V_{1}^{2} \omega^{4} S^{2} e^{-S}=10^{8} .
$$

Using the observed value of the spectral index, $n_{s}>0.97$ [13], we obtain a constraint from Eq. (17). Although $\Gamma$ is strictly constant in the present model, one could imagine that the parameters of the model vary slowly with $\varphi$ and thus provide a source of deviation from the Harrison-Zeldovich spectrum $n_{s}=1$ through $\dot{\Gamma}$. It is however unlikely that the two terms $4 \dot{H} / H^{2}$ and $\dot{\Gamma} / H \Gamma$ should be finely tuned against each other to give a small result. We therefore demand that $\left|4 \dot{H} / H^{2}\right|<0.04$ (the $68 \%$ c.l. limit). This leads to $A \Delta \varphi / V_{0}<5 \times 10^{-11}$. Using (19) and (22) to eliminate $A$ and $V_{0}$, this leads to an upper bound on $\Delta \varphi=2 \pi / \omega$, depending upon the effective self-coupling $\lambda=V_{1} \omega^{4}$,

$$
\log _{10}\left(\Delta \varphi / M_{p}\right)<-5.25-\frac{1}{2} \log _{10} \lambda-\frac{36.2}{\lambda}
$$

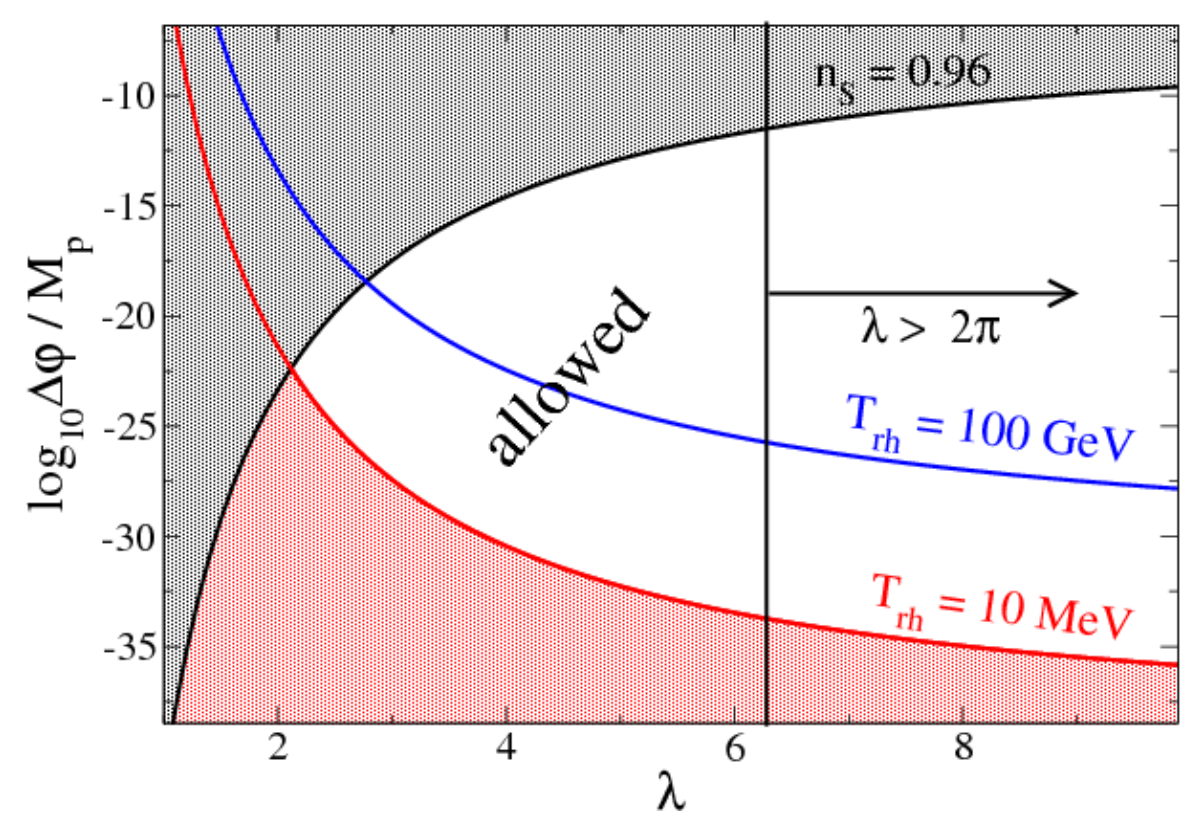

Figure 5: Unshaded regions in the $\lambda-\Delta \varphi$ plane are those for which the spectral index is not too red and the reheat temperature is above $10 \mathrm{MeV}$. The wedge-like region labeled "allowed" additionally fulfills the consistency requirement $\lambda<2 \pi$.

For our description in terms of nucleation of individual bubbles to be consistent, the bubbles must not be strongly overlapping. For the thick-walled bubbles we are 
considering, the initial radius of the bubble is of order the inverse mass scale, $r \sim M^{-1} \sim$ $\omega / \sqrt{\lambda}$. Since $\Gamma / H^{4}=10^{8}$, there must be room for $10^{8}$ such bubbles in a 4-dimensional Hubble volume: $10^{8} \times 2 \pi^{2} r^{4}<H^{-4}$. Using $3 M_{p}^{2} H^{2}=V_{0}$ and 22 to eliminate $V_{0}$, this becomes an upper limit on $\lambda$,

$$
2 \pi^{2} S^{2} e^{-S}<1
$$

which for the case $B=6$ is solved by $\lambda<49$. This of course is far weaker than the field theoretic consistency bound $\lambda<2 \pi$ discussed in section 2 .

One would like to ensure that the change in $\varphi$ remains less than $M_{p}$ over the course of the field's evolution. Assuming there were $N_{e}$ e-foldings of inflation, the total change in $\varphi$ implied by Eq. (8) is $\Delta^{\left(N_{e}\right)} \varphi=\left(4 \pi \Gamma / 3 H^{4}\right) \Delta \varphi N_{e}$ where $\Delta \varphi=2 \pi / \omega$ is the change in $\varphi$ between successive minima of the potential. We demand that $\Delta^{\left(N_{e}\right)} \varphi<M_{p}$ for $N_{e}=60$. This gives the upper bound $\Delta \varphi<10^{-10} M_{p}$, which is weaker than 23 and thus does not play an important role.

On the other hand, the requirement of a large enough reheating temperature $T_{\text {rh }}$ does give an interesting constraint on $\Delta \varphi$ that is complementary to (23). The reason is that $T_{\mathrm{rh}} \sim V_{0}^{1 / 4}$ and from $222 V_{0} \propto(\Delta \varphi)^{2}$, so low values of $\Delta \varphi$ as needed to satisfy (23) may also lead to excessively low reheat temperatures. If we generously assume such efficient reheating that $T_{\mathrm{rh}}=V_{0}^{1 / 4}$ and that $T_{\mathrm{rh}}>10 \mathrm{MeV}$ so that big bang nucleosynthesis can proceed normally, we obtain

$$
\log _{10}\left(\Delta \varphi / M_{p}\right)>-39.5+2 \log _{10}\left(\frac{T_{\mathrm{rh}}}{10 \mathrm{MeV}}\right)+\frac{36.2}{\lambda}
$$

We have summarized the most important constraints in figure 5, which plots them in the $\lambda-\Delta \varphi$ plane. If $T_{\mathrm{rh}}=10 \mathrm{MeV}$, there is an interval of $\lambda \in[2.1,2 \pi]$ where it is possible to find consistent models; at $T_{\mathrm{rh}}=100 \mathrm{GeV}$, more preferable for being able to generate a baryon asymmetry, the range shrinks to $\lambda \in[2.8,2 \pi]$ If one is willing to relax the field-theoretic consistency condition $\lambda<2 \pi$ for some reason, it becomes much easier to find viable models.

\section{Non-Gaussianities}

Unlike the case of perturbative quantum fluctuations from the vacuum, in chain inflation the perturbations come from light-cone shaped bubbles. Thus one might worry about the Gaussianity of the scenario. However, as there are a great number of bubbles per Hubble time and Hubble volume, the Poisson-distributed field fluctuation approaches a Gaussian distribution. Further, as we shall show, all the non-Gaussianity from $n$-point correlations turns out to be small.

The three-point correlation function can be calculated similarly to the case in Section 4. Note that for $X \sim \operatorname{Pois}(\mu),\left\langle(X-\langle X\rangle)^{3}\right\rangle=\mu$. We have

$$
\left\langle\delta \varphi^{3}\right\rangle=\Delta \varphi^{3} \int_{-\infty}^{\tau} \mathrm{d} \eta V_{3}(\eta) \frac{\Gamma}{H^{4} \eta^{4}},
$$


where $V_{3}(\eta)$ is the volume of the intersection region of three balls with radius $\tau-\eta$. Writing in terms of $\zeta$, and comparing to the definition of $f_{N L}$ [14,

$$
\left\langle\zeta^{3}\right\rangle=(2 \pi)^{3} \delta^{3}\left(\mathbf{k}_{1}+\mathbf{k}_{2}+\mathbf{k}_{3}\right) \frac{3}{5} f_{N L} P_{\zeta}^{2},
$$

we find $f_{N L}=\mathcal{O}(1)$.

Higher point correlations can be estimated in the same way. For $X \sim \operatorname{Pois}(\mu),\langle(X-$ $\left.\langle X\rangle)^{4}\right\rangle=\mu+3 \mu^{2},\left\langle(X-\langle X\rangle)^{5}\right\rangle=\mu+10 \mu^{2},\left\langle(X-\langle X\rangle)^{6}\right\rangle=\mu+25 \mu^{2}+15 \mu^{3}$. For the fourpoint function, the $3 \mu^{3}$ term must be subtracted to remove the disconnected part. Thus the connected part has $\left\langle(X-\langle X\rangle)^{4}\right\rangle_{c}=\mu$. In the language of non-Gaussian estimators, this corresponds to $g_{N L}=\mathcal{O}(1)$. Similarly, the five- and six-point estimators $h_{N L}$ and $i_{N L}$ defined in [15] are also of order one. This statement generalizes to arbitrary $n$-point correlation functions. Thus non-Gaussianity for single-field chain inflation is small at any order.

\section{Generalizations}

In this section we briefly discuss three generalizations of single-field chain inflation, namely the curvaton scenario, the multi-field case, and a noncanonical kinetic term.

We start with the curvaton scenario, where there is a second field whose rolling (or other dynamics) dominates the change in the total energy density. In this case, the fluctuation in the chain tunneling direction (still denoted by $\varphi$ here) is an isocurvature perturbation. This isocurvature perturbation converts to a curvature perturbation later by oscillation of $\varphi$ around its minimum, or if the chain of tunnelings ends after the reheating associated with the inflaton. Suppose there is no direct interaction (except via gravity) between the inflaton sector and the $\varphi$ field. Then the component curvature perturbation $\zeta_{\varphi}$ is conserved, which is related to the total curvature perturbation $\zeta$ as

$$
\zeta=r \zeta_{\varphi}, \quad r=\frac{\rho_{\varphi}+p_{\varphi}}{\rho+p},
$$

where $\rho, p, \rho_{\varphi}, p_{\varphi}$ are the total energy density and pressure and the energy density and pressure for the $\varphi$ field respectively. $\zeta_{\varphi}$ is related to the field fluctuation as $\zeta_{\varphi}=$ $-H \delta \varphi / \dot{\varphi}$. After the curvaton $\varphi$ decays, $r$ becomes constant, thus $\zeta$ is subsequently conserved. The power spectrum can be calculated similarly to the last section as

$$
P_{\zeta}=\frac{3 r^{2} H^{4}}{4 \pi \Gamma} .
$$

Since it is suppressed by $r^{2}$, the original fluctuation in the $\varphi$ field may be large. This reduces the number of minima needed in the $\varphi$ potential, but at the same time it increases the non-Gaussianity $f_{N L}=\mathcal{O}(1 / r)$, which should be the best estimator for non-Gaussianity, and is potentially observable.

It is also interesting to generalize the simplest scenario to a multi-field case, where a chain of tunnelings can take place in more than one field direction. Whenever successive 
tunnelings occur in orthogonal field-space directions, the collision of bubble walls will not trigger a new phase nucleation.

In this case, for a given nucleation rate $\Gamma$, an individual observer will undergo fewer phase transitions. Specifically, the mean inter-bubble spacing is $\delta r \sim \Gamma^{-1 / 4}$, the mean time between phase changes for a comoving observer is $\delta t \sim \delta r$, and so the mean number of phase changes per Hubble time is $\sim\left(\Gamma / H^{4}\right)^{1 / 4}$, not $\sim \Gamma / H^{4}$ as we found for the singlefield case.

Perturbations in this scenario remain correlated on scales shorter than the Hubble scale. The reason is that, while individual bubble walls no longer propagate Hubble scale distances, information about bubble nucleations still can. As an illustration, consider figure 6. In the figure, one region of space (near $A$ ) reaches $\varphi=\varphi_{0}$ before another region (near $B$ ). But the probability that $B$ will encounter another phase change and get to $\varphi_{0}+\Delta \varphi$ is determined by the likelihood of a nucleation occurring in the shaded area in its past light cone. This region is larger, and hence the chance of a nucleation is larger, than the shaded area in A's past light cone. Therefore, unevennesses in the phase boundary allows regions which are "behind" to "catch up," at least on sub-Hubble scales. This means that perturbations from the stochastic nature of nucleations get averaged over roughly Hubble-scale volumes.

However, on super-Hubble scales no such communication is possible. Therefore the progress of the field $\varphi$ on super-Hubble scales is still determined by the independent, Poisson-distributed number of nucleations in the past light cone. Hence we estimate that the power spectrum is again $P_{\zeta}=\left(H^{4} / \Gamma\right)$, similar to the power spectrum for single-field chain inflation. We have not been able to evaluate the order-1 coefficient analytically in this case. But this is enough to show that the tunneling rate must be comparable to that in the single-field case, though the number of minima in the potential can be substantially smaller $\left(\sim 10^{4}\right.$ rather than $\left.\sim 10^{10}\right)$.

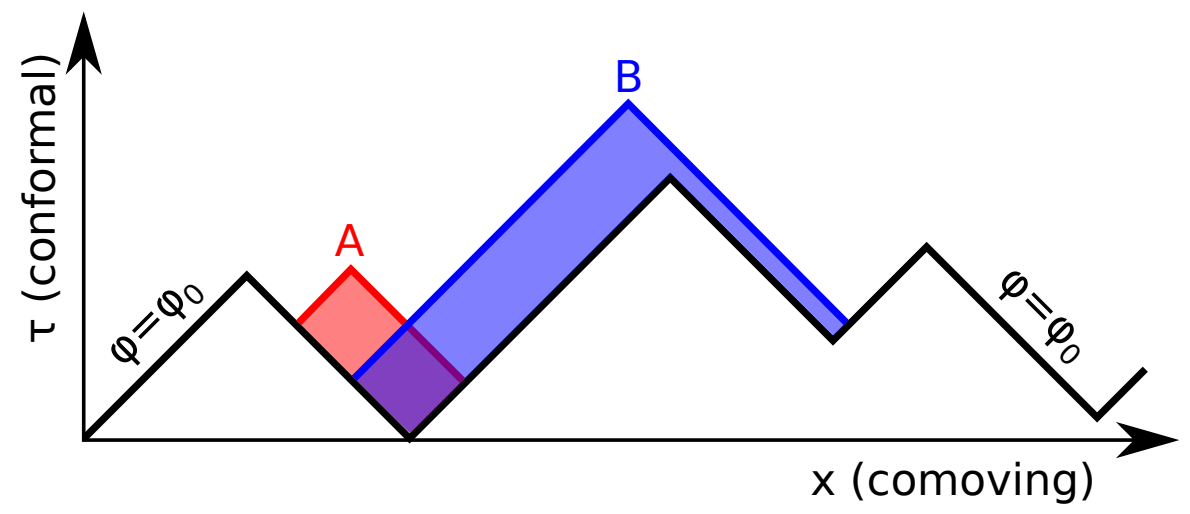

Figure 6: The probability for $B$ in the next step of the chain is larger than that of $A$, even if bubble wall collision no longer triggers a new bubble nucleation.

A third possible generalization of chain inflation is to allow for a noncanonical kinetic term. There is some motivation to do so since ref. [16] pointed out that the tunneling rate in a generic scalar field theory with false minima can be increased using the string- 
motivated DBI action with Lagrangian

$$
\mathcal{L}=-\frac{1}{f(\varphi)}\left(\sqrt{1-(\partial \varphi)^{2} f(\varphi)}-1\right)-V(\varphi)
$$

If $f$ is a constant, the tunneling action can be reduced by at most a factor of order unity; to achieve a greater reduction, one must choose $f$ to be proportional to the term $V_{1} \sin (\omega \varphi)$ that gives the barrier, and tune $f V_{1} \rightarrow 2 . S$ is then reduced by the factor $\left(1-f V_{1} / 2\right)^{2}$. However taking $f \propto V_{1} \sin (\omega \varphi)$ seems quite contrived, so we consider to what extent the modest reduction in $S$ afforded by constant $f$ can help to alleviate the tension between various constraints discussed in section 5 .

It is amusing to notice that the tunneling action $S$ can still be computed exactly within the thin-wall approximation (even though we did not use this approximation in our previous analysis) for the model of section 5 and the DBI action with constant $f$. In the thin-wall approximation, $S$ is reduced relative to its usual value by the factor $\frac{1}{2}\left(1+\frac{1-a}{2 \sqrt{a}} \ln \frac{1+\sqrt{a}}{1-\sqrt{a}}\right)$ where $a=f V_{1} / 2$. When $a=0$ we recover the usual result, and when $a=1$ we obtain the maximum possible reduction in $S$ for constant $f$, a factor of $1 / 2$. Let us take this as indicative of how well one might hope to do in the case of interest where the thin-wall approximation is not valid. (We are not enthusiastic enough about this generalization to do a full numerical solution of the bubbles.) The most important constraints, those on the spectral index and the reheat temperature, are both relaxed since the spectral index bound goes like $\Delta \varphi<\# S^{1 / 2} e^{-S / 4}$ while the reheating bound has the form $\Delta \varphi>\# S^{-1 / 2} e^{S / 4}$. We find that the minimum allowed value of $\lambda$ decreases to 1.1 for $T_{\mathrm{rh}}=10 \mathrm{MeV}$, and to 1.4 for $T_{\mathrm{rh}}=100 \mathrm{GeV}$. The model remains rather strongly coupled, but less so than for a standard kinetic term.

\section{Conclusion}

To conclude, we reconsidered models of chain inflation focusing on the single-field case, and giving what we consider to be the first correct derivation of the spectrum of fluctuations, taking into account their Poisson nature as a result of the dynamics of bubble collisions. We showed that the power spectrum can be calculated analytically and that consistency with the size of density perturbations measured today requires $10^{8}$ tunnelings per Hubble volume per e-fold. Therefore single-field models of chain inflation need a potential with at least $O(60) \times 10^{8} \sim 10^{10}$ successive minima, a significant challenge for model building. The spectral tilt depends on details of the potential; it can be either red or blue and need not be small. Non-Gaussianities in the model are small, of order unity. Thermal contributions to the fluctuations are negligible in this scenario because the energy stored in bubble walls remains in them after they collide.

A challenge for chain inflation is posed by a standard requirement of consistency for any field theory. Under renormalization, couplings run, and large couplings run very fast. In order to have any nonnegligible range of scales over which the theory remains valid, the effective coupling given by the fourth derivative of the potential must not be much larger than $O(1)$. We have shown that this requirement, together with constraints on 
the spectral index and reheating temperature, leaves only a small interval of parameter space that gives a large enough nucleation rate.

On the other hand, the method used to calculate the power spectrum and nonGaussianity in this model may find application for cases where other discrete processes instead of a continuous process take over the role of quantum fluctuations during inflation - for example, if the scale of inflation is very high and physics becomes discrete near the Planck scale.

\section{Acknowledgment}

We thank Bret Underwood for discussion. YW is supported by grants from McGill University, Fonds Québécois de la Recherche sur la Nature et les Technologies (FQRNT), the Institute of Particle Physics (Canada) and the Foundational Questions Institute. This work was supported in part by the Natural Sciences and Engineering Research Council of Canada.

\section{References}

[1] K. Freese, D. Spolyar, "Chain inflation: 'Bubble bubble toil and trouble'," JCAP 0507, 007 (2005). hep-ph/0412145.

[2] K. Freese, J. T. Liu, D. Spolyar, "Inflating with the QCD axion," Phys. Rev. D72, 123521 (2005). [hep-ph/0502177].

[3] A. H. Guth, "The Inflationary Universe: A Possible Solution to the Horizon and Flatness Problems," Phys. Rev. D 23, 347 (1981).

[4] B. Feldstein, B. Tweedie, "Density Perturbations in Chain Inflation," JCAP 0704, 020 (2007). hep-ph/0611286.

[5] D. Chialva, U. H. Danielsson, "Chain inflation revisited," JCAP 0810, 012 (2008). arXiv:0804.2846 [hep-th]].

[6] D. Chialva, U. H. Danielsson, "Chain inflation and the imprint of fundamental physics in the CMBR," JCAP 0903, 007 (2009). arXiv:0809.2707 [hep-th]].

[7] Q. -G. Huang, "Simplified chain inflation," JCAP 0705, 009 (2007). arXiv:0704.2835 [hep-th]].

[8] S. R. Coleman, "The Fate of the False Vacuum. 1. Semiclassical Theory," Phys. Rev. D15, 2929-2936 (1977).

[9] S. R. Coleman, F. De Luccia, "Gravitational Effects on and of Vacuum Decay," Phys. Rev. D21, 3305 (1980). 
[10] R. Easther, J. T. Giblin, L. Hui and E. A. Lim, "A New Mechanism for Bubble Nucleation: Classical Transitions," Phys. Rev. D 80, 123519 (2009) arXiv:0907.3234 [hep-th]]; J. T. Giblin, L. Hui, E. A. Lim and I. S. Yang, "How to Run Through Walls: Dynamics of Bubble and Soliton Collisions," Phys. Rev. D 82, 045019 (2010) arXiv:1005.3493 [hep-th]].

[11] A. Ashoorioon, K. Freese and J. T. Liu, "Slow nucleation rates in Chain Inflation with QCD Axions or Monodromy," Phys. Rev. D 79, 067302 (2009) arXiv:0810.0228 [hep-ph]]; A. Ashoorioon and K. Freese, "Gravity Waves from Chain Inflation," arXiv:0811.2401 [hep-th].

[12] C. G. Callan, Jr., S. R. Coleman, "The Fate of the False Vacuum. 2. First Quantum Corrections," Phys. Rev. D16, 1762-1768 (1977).

[13] E. Komatsu et al. [ WMAP Collaboration ], "Seven-Year Wilkinson Microwave Anisotropy Probe (WMAP) Observations: Cosmological Interpretation," Astrophys. J. Suppl. 192, 18 (2011). arXiv:1001.4538 [astro-ph.CO]].

[14] E. Komatsu, D. N. Spergel, "Acoustic signatures in the primary microwave background bispectrum," Phys. Rev. D63, 063002 (2001). astro-ph/0005036.

[15] C. Lin, Y. Wang, "Quadra-Spectrum and Quint-Spectrum from Inflation and Curvaton Models," JCAP 1007, 011 (2010). arXiv:1004.0461 [astro-ph.CO]].

[16] A. R. Brown, S. Sarangi, B. Shlaer, A. Weltman, "A Wrinkle in Coleman-De Luccia," Phys. Rev. Lett. 99, 161601 (2007). arXiv:0706.0485 [hep-th]]. 